Case Report

\title{
A Unique Case of Relapsing Polychondritis Presenting with Acute Pericarditis
}

\author{
John V. Higgins, ${ }^{1}$ Uma Thanarajasingam, ${ }^{2}$ and Thomas G. Osborn ${ }^{2}$ \\ ${ }^{1}$ Department of Medicine, Mayo Clinic, 200 1st Street SW, Rochester, MN 55905, USA \\ ${ }^{2}$ Division of Rheumatology, Department of Medicine, Mayo Clinic, Rochester, MN 55905, USA
}

Correspondence should be addressed to John V. Higgins; higgins.john@mayo.edu

Received 10 October 2013; Accepted 26 November 2013

Academic Editors: R. Cevik, J. Mikdashi, J. C. Nossent, and M. Soy

Copyright @ 2013 John V. Higgins et al. This is an open access article distributed under the Creative Commons Attribution License, which permits unrestricted use, distribution, and reproduction in any medium, provided the original work is properly cited.

Relapsing polychondritis (RP) is an inflammatory disease of the cartilaginous tissue primarily affecting the cartilaginous structures of the ear, nose, joints, and the respiratory system. Cardiovascular complications of RP are associated with high morbidity and mortality and occur most commonly as valvular disease. Pericarditis is a less common complication, occurring in $4 \%$ of patients with RP and has not previously been described at presentation. We describe a case of relapsing polychondritis with acute pericarditis at presentation.

\section{Introduction}

Relapsing polychondritis (RP) is an autoimmune disorder of unknown etiology primarily affecting the cartilaginous structures of the body. It may also involve other noncartilaginous, proteoglycan rich organs [1]. It is a relatively rare disease with no animal model, making investigation into the mechanism of disease more difficult. Due to this, RP is often difficult to diagnose and treat, with life threatening consequences, if proper diagnosis is not made [2]. The disease is episodic and progressive, with a heterogenous phenotype [3]. The most common presenting features include auricular chondritis, seronegative arthritis, nasal chondritis, ocular inflammation, and laryngotracheal symptoms $[1,3]$.

Infection and respiratory problems are the most common cause of death, but cardiac complications are the next most common cause of mortality [4]. Valvular involvement is the most common cardiac cause of both morbidity and mortality. Other less common cardiac complications include conduction disturbances, pericarditis, vasculitis, and vascular disease such as aortic aneurysm or dissection [5]. Cardiovascular complications almost universally present later in the disease course with a mean interval of six years after presentation [5]. Only AV nodal conduction abnormalities, presenting as a third degree heart block, have been reported in the literature at presentation of RP [6]. Acute pericarditis is a relatively rare complication of RP, present in only $4 \%$ of patients during the course of the disease, and has never been reported at the time of presentation [5]. We present the case of a patient diagnosed with RP who was found to have acute pericarditis on presentation.

\section{Case Report}

A 31-year-old woman presented to the emergency department with chest and facial pain. She had no significant past medical history. She was in her usual state of health until 6 weeks prior to presentation when she developed rhinitis and cough. She had been treated with antibiotics with no relief. She then developed pain and swelling on the bridge of her nose, bilateral cheeks, and bilateral eyelids. She was treated with a brief course of corticosteroids with mild relief in symptoms. She was evaluated by an otolaryngologist 2 weeks before presentation; she was given a diagnosis of facial cellulitis and was restarted on antibiotic therapy.

Four weeks prior to presentation, she developed migratory pleuritic-type chest pain and associated tenderness to palpation over the areas of pain. Her symptoms improved 


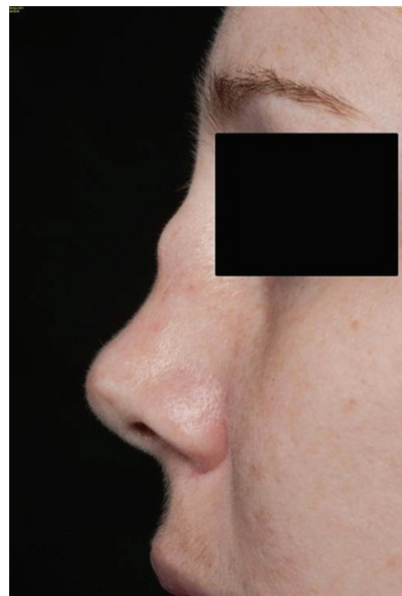

FIGURE 1: Acute onset saddle nose deformity due to inflammation of the nasal cartilage demonstrated six weeks following symptom onset.

in the upright position. Her chest and facial pain became intolerable, and she presented for further evaluation.

On physical exam, she was noted to have a saddle nose deformity with edema of the nasal mucosa (Figure 1) and pain to palpation over the costal cartilage of the right 4-5th ribs. There was no auricular inflammation, tracheal tenderness, or synovitis. Cardiac exam was normal without cardiac rub. Systems review was negative for nasal crusting or epistaxis, diminished hearing or vision, paresthesias, or any sensory loss.

Initial laboratory data was remarkable for a sedimentation rate of $46 \mathrm{~mm} / \mathrm{hr}(0-29 \mathrm{~mm} / \mathrm{hr})$, C-reactive protein of $104.4 \mathrm{mg} / \mathrm{L}(<8 \mathrm{mg} / \mathrm{L})$, hemoglobin of $10.7 \mathrm{~g} / \mathrm{dL}(12.0-15.5)$, and a mean corpuscular volume of $88.6 \mathrm{fL}$ (81.6-98.3 fL). Antineutrophil cytoplasmic antibodies (ANCAs), antinuclear antibodies, rheumatoid factor, and creatinine were all unremarkable. Influenza, respiratory syncytial virus, and human immunodeficiency virus studies were negative.

Computed tomography (CT) scan of the chest was performed with contrast and revealed a nonspecific, ground glass nodular infiltrate of the right lower lobe (Figure 2). A transthoracic echocardiogram (TTE) revealed a pericardial effusion around the right atrium with basal inferior and inferoseptal hypokinesis without valvular disease. A subsequent cardiac MRI showed pericardial enhancement over the right ventricular free wall consistent with acute pericarditis (Figure 3). Ophthalmologic exam was negative for uveitis or other pathology. Given the findings of nasal chondritis, acute noninfectious pericarditis, nonspecific ground glass opacities by CT scan possibly secondary to inflammation, and elevated inflammatory markers, as well as the lack of serologic or clinical findings for ANCA-associated vasculitis, a clinical diagnosis of RP was made.

She was started on prednisone $30 \mathrm{mg}$ daily, colchicine $0.6 \mathrm{mg}$ daily, and dapsone $50 \mathrm{mg}$ daily. She was then dismissed from the hospital with improvement in her symptoms.

Approximately 8 weeks following discharge, while tapering prednisone to $20 \mathrm{mg}$ daily, she had recurrence of facial

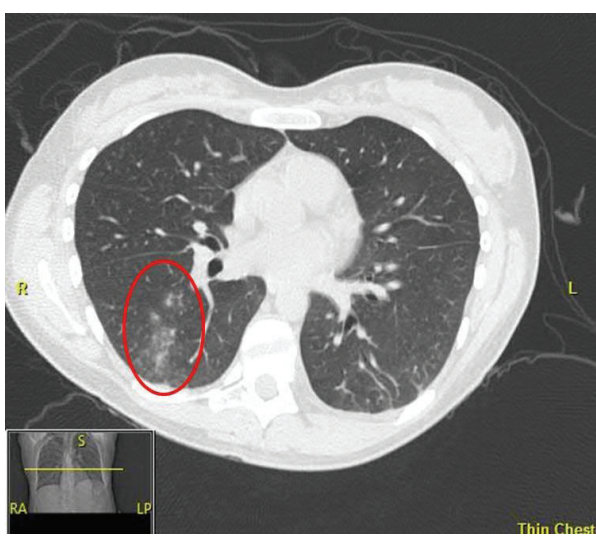

FIGURE 2: Ground glass opacities in the right lower lobe consistent with an acute inflammatory process.

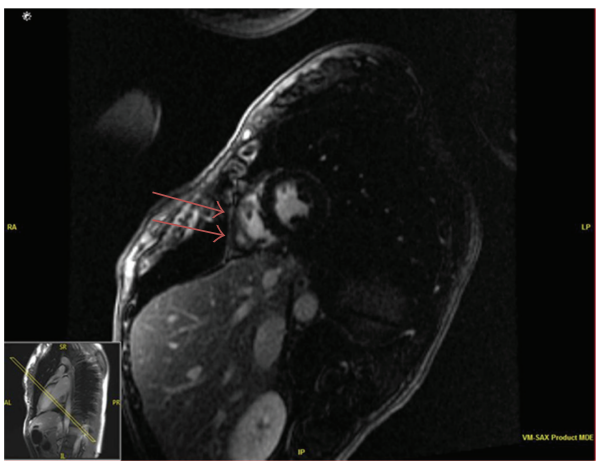

Figure 3: Pericardial enhancement of the right ventricular free wall on cardiac MRI consistent with acute pericarditis.

pain and swelling and developed auricular inflammation. Bilateral nasal biopsies were performed to rule out vasculitis. This was notable for mild to moderate inflammation, with no granulomas or evidence of vasculitis. Her prednisone dose was increased, and symptoms resolved. She continues on dapsone and a prednisone taper with plans to undergo nasal reconstructive surgery in the upcoming year. She has had no recurrence of chest pain.

\section{Discussion}

The diagnosis of relapsing polychondritis is typically clinical; there is no specific serologic test for RP [3]. The McAdams criteria were the initial diagnostic criteria [7] and required three out of six of the following: bilateral auricular chondritis, nonerosive seronegative inflammatory arthritis, nasal chondritis, ocular inflammation, respiratory tract chondritis, and audiovestibular damage. Modified criteria have been proposed by Damiani and Levine [8] which include having 1 McAdam criterion plus tissue diagnosis or 2 McAdam criteria plus response to corticosteroids. The Michet criteria include proven inflammation in 2 of 3 areas-auricular, nasal, or 
laryngotracheal cartilages-as well as two of the following: ocular inflammation, vestibular dysfunction, seronegative arthritis, and hearing loss [4]. This patient had nasal chondritis, acute pericarditis, and likely respiratory chondritis, as well as a robust response to corticosteroids. Additionally, her relapse of severe inflammation of the nasal cartilage and ear discomfort during the initial prednisone taper supports the diagnosis as well.

Cardiovascular complications are responsible for approximately $18 \%$ of deaths in RP patients trailing only pulmonary and infectious causes of death [9]. These can occur at any point in the disease course. With advanced diagnostic techniques, pericarditis may be a more frequently recognized component of RP. It is important to differentiate between RP and other connective tissue diseases as they have variable complications and disease courses. Due to the potential for these life threatening complications, cardiac surveillance with echocardiogram should be performed at all stages of disease, including presentation [9]. Potential serious complications such as atrial flutter have been associated with acute pericarditis in RP [10].

Immunosuppressive therapy is the mainstay of treatment for RP for both active disease and cardiac complications. Prednisone is the traditional mainstay of therapy with nonsteroidal anti-inflammatory drugs and dapsone for milder disease. Cyclosporine A and cyclophosphamide are typically reserved for refractory disease and organ threatening damage [1]. Active flares including cardiac complications have been reported to occur with suspension of therapy [11].

Cardiovascular complications which are not amenable to treatment with medical therapy may require further intervention to treat the underlying problem. Pacemaker implantation is required in patients who develop high grade AV block [9]. Additionally, patients who develop severe valvular regurgitation causing symptomatic heart failure require surgical repair. Aortic valve involvement is more common than mitral valve involvement, with no reported complications of the tricuspid or pulmonary valves [9]. In addition, aortic root dilation may occur, leading to aortic regurgitation in $10 \%$ of patients [12]. This may require surgical replacement. When pericarditis has been reported in RP, it has not been associated with hemodynamic compromise, but it should be treated appropriately with nonsteroidal antiinflammatory drugs and/or colchicine. Pulmonary disease was also evident in our patient with nonspecific inflammation and ground glass opacities shown on CT of the chest. Inflammatory airway edema is the likely inciting factory for lower respiratory tract disease in RP and this precedes the more worrisome respiratory finding of airway stenosis [13].

This case highlights a rare presenting sign of relapsing polychondritis. Cardiac complications should be monitored at all stages of the disease course with appropriate intervention to prevent further, potentially fatal consequences.

\section{Disclosure}

This paper has not been submitted or published elsewhere.

\section{References}

[1] P. D. Kent, C. J. Michet Jr., and H. S. Luthra, "Relapsing polychondritis," Current Opinion in Rheumatology, vol. 16, no. 1, pp. 56-61, 2004.

[2] D. E. Trentham and C. H. Le, "Relapsing polychondritis," Annals of Internal Medicine, vol. 129, no. 2, pp. 114-122, 1998.

[3] E. Letko, P. Zafirakis, S. Baltatzis, A. Voudouri, C. Livir-Rallatos, and C. S. Foster, "Relapsing polychondritis: a clinical review," Seminars in Arthritis and Rheumatism, vol. 31, no. 6, pp. 384395, 2002.

[4] C. J. Michet Jr., C. H. McKenna, H. S. Luthra, and W. M. O'Fallon, "Relapsing polychondritis: survival and predictive role of early disease manifestations," Annals of Internal Medicine, vol. 104, no. 1, pp. 74-78, 1986.

[5] C. Maineguene, J. B. Bouhour, A. Y. de Lajartre et al., "Les complications cardiovasculalres de la polychondrite chronique atrophiante: apropos d'un cas anatomo-clinique. Revue de la litterature," Annales de Cardiologie et d'Angéiologie, vol. 40, pp. 97-102, 1991.

[6] B. Hojaili and H. D. Keiser, "Relapsing polychondritis presenting with complete heart block," Journal of Clinical Rheumatology, vol. 14, no. 1, pp. 24-26, 2008.

[7] L. P. McAdam, M. A. O'Hanlan, R. Bluestone, and C. M. Pearson, "Relapsing polychondritis: prospective study of 23 patients and a review of the literature," Medicine, vol. 55, no. 3, pp. 193-215, 1976.

[8] J. M. Damiani and H. L. Levine, "Relapsing polychondritis. Report of ten cases," Laryngoscope, vol. 89, no. 6, pp. 929-946, 1979.

[9] A. Del Rosso, N. R. Petix, M. Pratesi, and A. Bini, "Cardiovascular involvement in relapsing polychondritis," Seminars in Arthritis and Rheumatism, vol. 26, no. 6, pp. 840-844, 1997.

[10] C. Dapogny, G. Grollier, J. H. Bertrand et al., "Les manifestations cardiaques de la polychondrite atrophiante: a propos d'uncas se manifestant par un epanchement pericardique et un flutter anriculaire," Annales de Cardiologie et d'Angéiologie, vol. 34, pp. 621-624, 1985.

[11] M. C. Mayer, M. Visconti, P. Bassano, and V. Galloro, "La policondrite ricorrente: descrizione di un caso con peculiar interessamento cardiaco," Recenti Progressi in Medicina, vol. 82, pp. 83-85, 1991.

[12] S. N. Barretto, G. H. Oliveira, C. J. Michet Jr., M. A. Nyman, W. D. Edwards, and I. J. Kullo, "Multiple cardiovascular complications in a patient with relapsing polychondritis," Mayo Clinic Proceedings, vol. 77, no. 9, pp. 971-974, 2002.

[13] S. Rafeq, D. Trentham, and A. Ernst, "Pulmonary manifestations of relapsing polychondritis," Clinics in Chest Medicine, vol. 31, no. 3, pp. 513-518, 2010.

\section{Conflict of Interests}

The authors declare that they have no conflict of interests. 


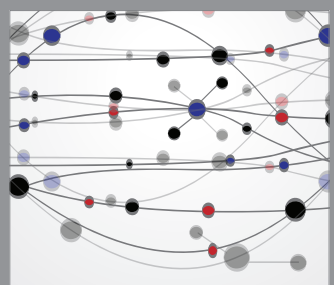

The Scientific World Journal
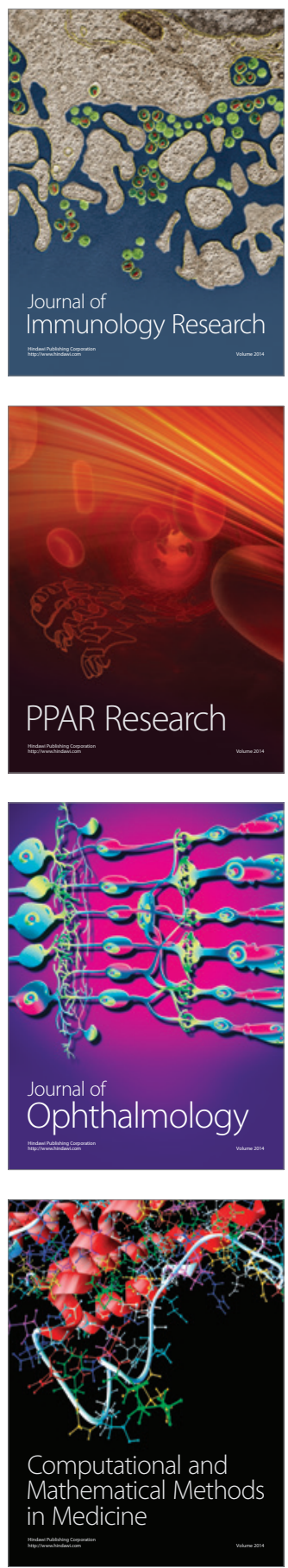

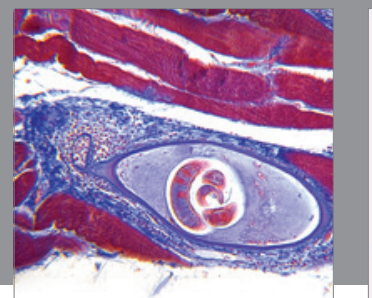

Gastroenterology

Research and Practice
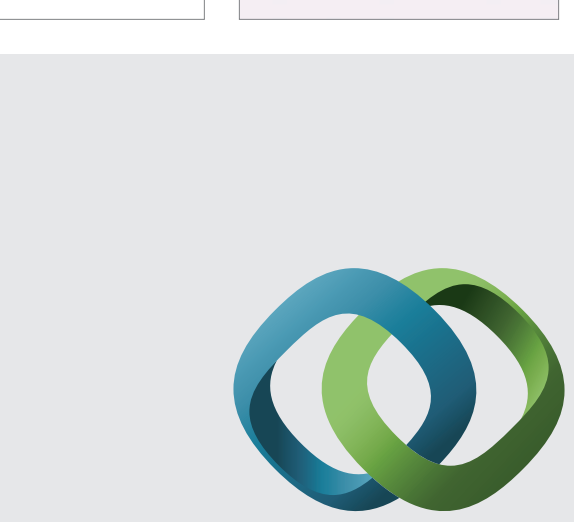

\section{Hindawi}

Submit your manuscripts at

http://www.hindawi.com
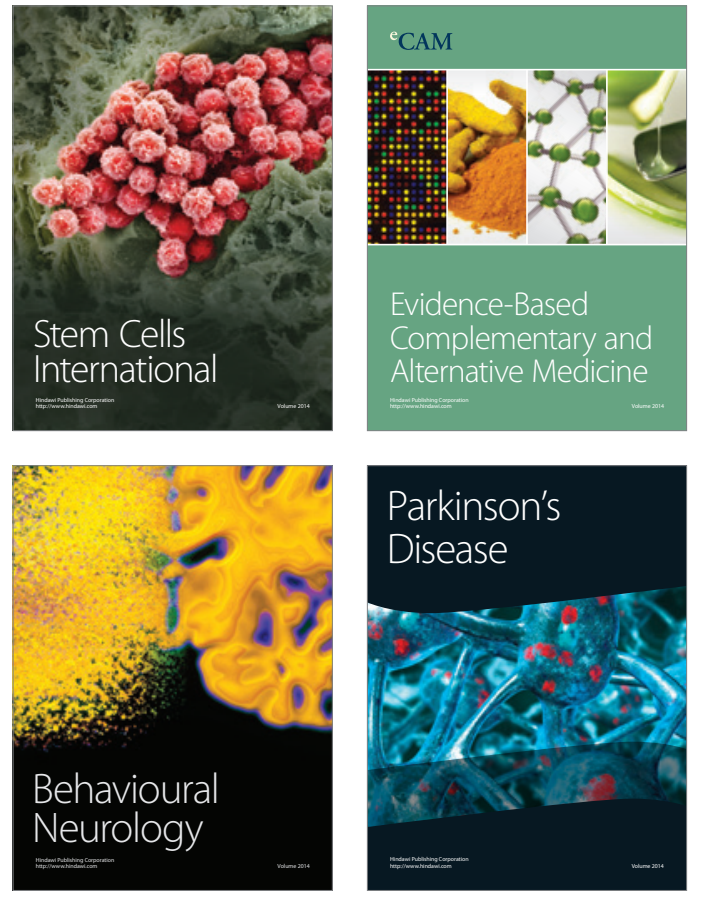
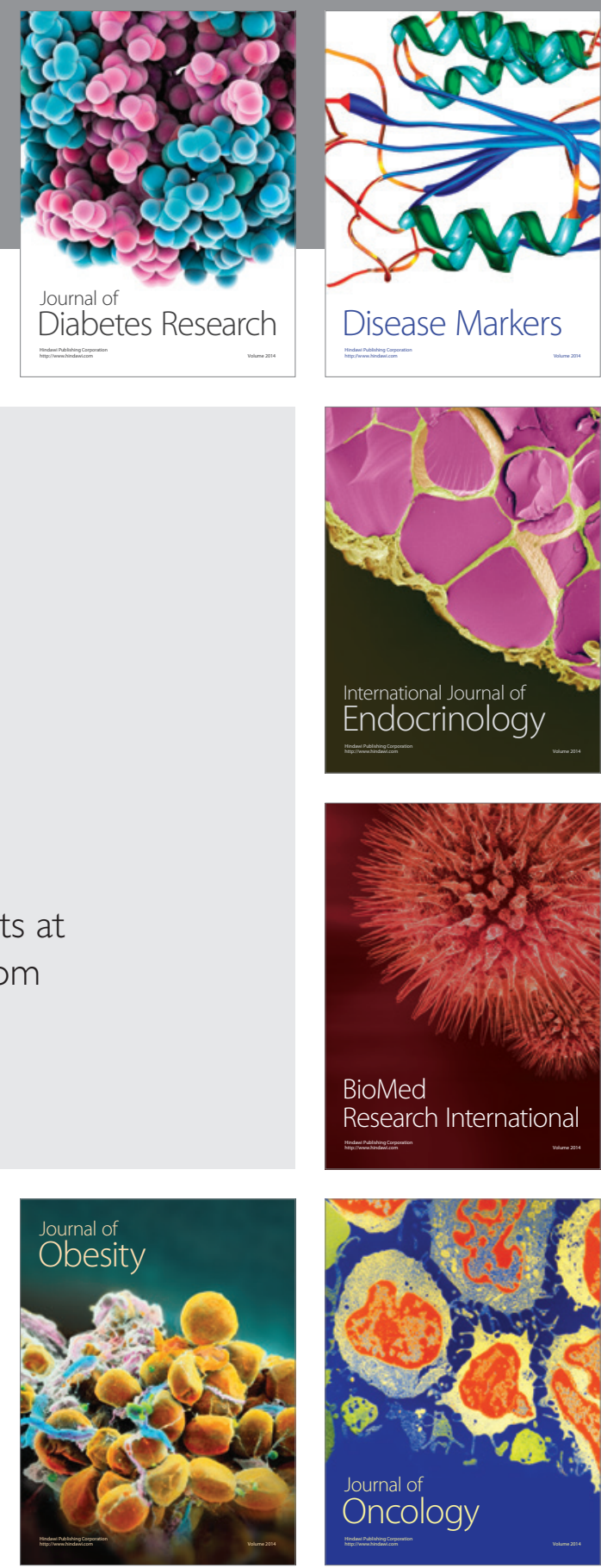

Disease Markers
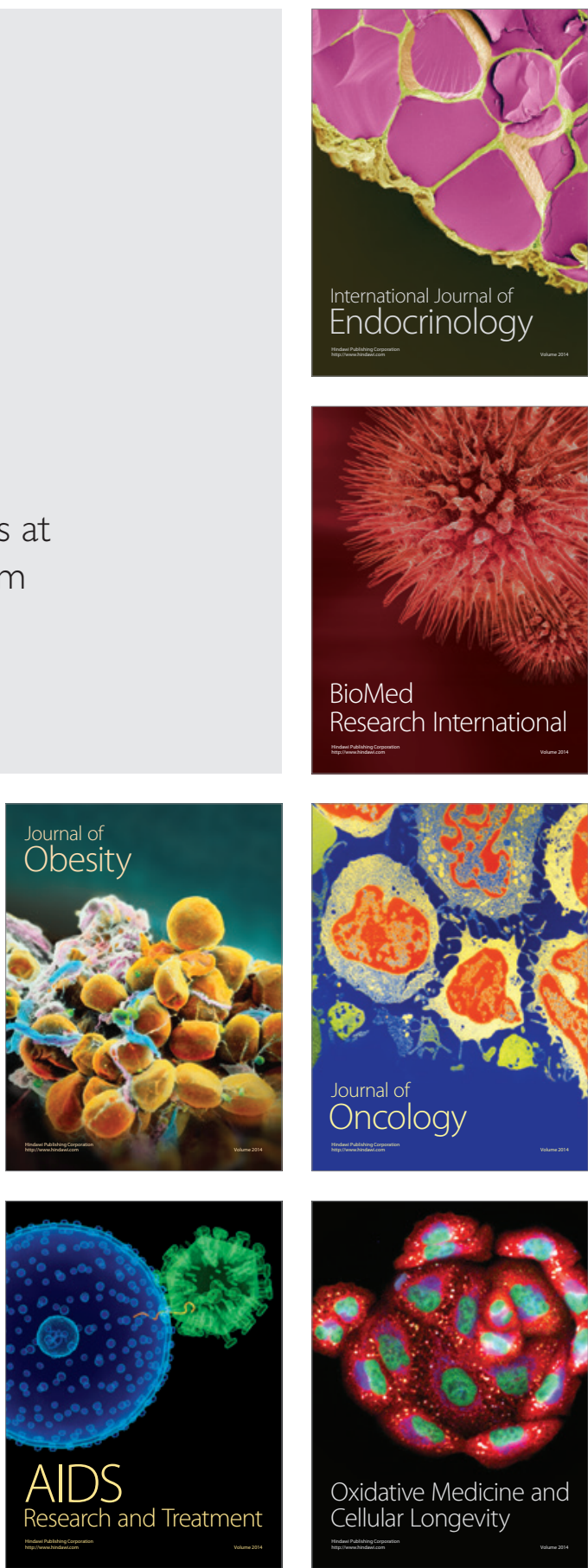